\title{
Calculation of the movement trajectory of the grain mass in the field stripper
}

\author{
Andrey Matrosov ${ }^{1}$, Daria Nizhnik ${ }^{1}$, Ivan Panfilov ${ }^{1}$, Viktor Pakhomov ${ }^{1,2}$,Irina \\ Serebryanaya ${ }^{1}$, Arkady Soloviev ${ }^{1, *}$, and Dmitriy Rudoy ${ }^{1}$ \\ ${ }^{1}$ Don State Technical University, 344003, pl. Gagarina, 1, Rostov-on-Don, Russia \\ ${ }^{2}$ State Scientific Establishment «Agricultural research center «Donskoy», 347740, Scientific town, 3, \\ Zernograd, Russia
}

\begin{abstract}
The work is devoted to the study of the dynamics of the grain mass in a field stripper for grain separation. At the first stage, a model of the movement of the air mass in the considered installation is built and based on a mathematical model that takes into account the turbulence of the movement of the air mass, using the method of finite volumes in the ANSYS package, the field of velocities and pressures is calculated. At the second stage, the movement of a fragment of the grain mass in this flow is considered, its trajectory is built taking into account the interaction with the upper deck of the installation. The performed calculations allow us to choose rational geometric and kinematic parameters at which no stagnant zones appear in the chamber, the grain mass interacts with the upper one, which contributes to the release of grain in the installation chamber.
\end{abstract}

\section{Introduction}

The urgent task of today in the grain sector of agriculture is the task of ensuring low-energy and low-traumatic forced extraction of grain from the ear. This requires not only the improvement of existing methods and methods of grain harvesting [1-7], but also the development of new highly efficient physical and mechanical methods of its harvesting and, accordingly, the design of the appropriate equipment [8-11].

The solution of this problem, on the one hand, requires the development and creation of adequate analytical and numerical physical and mechanical models, including models based on CAD-CAE complexes, capable of describing the interactions between the elements of the stem-ear-grain system. At the same time, on the other hand, - the development and creation of physical and mechanical models of the process of impact on the stem-ear-grain system of the working bodies of grain harvesting equipment.

In the previous work [12], the modes of action on the ear were studied, in which it is possible to extract grain from it due to the resonant movements of the ear and grain in the ear. This work is devoted to the study of the dynamics of the grain mass in a field stripper, which implements stripping harvesting of ears with further separation of grain and chaff.

The study of the movement of the grain mass can be divided into two steps using an iterative scheme for finding rational parameters. At the first stage, a model of the air mass

*Correcting author: solovievarc@gmail.com 
movement in the considered installations is built and based on a mathematical model that takes into account the turbulence of the movement, the velocity and pressure field is calculated using the finite volume method. At the second stage, a fragment of the grain mass in the installation chamber is considered and its trajectory is studied based on the integration of the equations of dynamics. The zones and speeds of its interaction with the body of the installations and the modes of vibration impact when sliding along the ribbed profile are determined. In the iterative process, on the basis of these data, the kinematic and geometric parameters of the installation are changed and the velocity field is re-calculated and the process is repeated until the required parameters are achieved. The use of an iterative algorithm allows solving an optimization problem. In this work, the main attention is paid to the second stage of the study. A series of calculations was carried out, the geometric and kinematic parameters of the installation were obtained, at which there are no stagnant zones in the chamber, the grain mass interacts with the upper one, which contributes to the release of grain in the installation chamber.

\section{Mathematical formulation of the problem of movement of grain mass in the installation.}

\subsection{Mathematical model of the movement of air mass}

Using $\kappa-\varepsilon$ - turbulence model [13-20] the mathematical model of the medium flow has the following form:

$$
\begin{gathered}
\frac{\partial\left(\rho V_{x}\right)}{\partial x}+\frac{\partial\left(\rho V_{y}\right)}{\partial y}=0 \\
\frac{\partial}{\partial x}\left(\rho V_{x} V_{x}\right)+\frac{\partial}{\partial y}\left(\rho V_{x} V_{y}\right)=-\frac{\partial \rho}{\partial x}+\frac{\partial}{\partial x}\left(\mu_{e} \frac{\partial V_{x}}{\partial x}\right)+\frac{\partial}{\partial y}\left(\mu_{e} \frac{\partial V_{x}}{\partial y}\right) \\
\frac{\partial}{\partial x}\left(\rho V_{x} V_{y}\right)+\frac{\partial}{\partial y}\left(\rho V_{y} V_{y}\right)=-\frac{\partial \rho}{\partial y}+\frac{\partial}{\partial x}\left(\mu_{e} \frac{\partial V_{y}}{\partial x}\right)+\frac{\partial}{\partial y}\left(\mu_{e} \frac{\partial V_{y}}{\partial y}\right) \\
\frac{\partial}{\partial x}\left(\rho V_{x} \kappa\right)+\frac{\partial}{\partial y}\left(\rho V_{y} \kappa\right)=\frac{\partial}{\partial x}\left(\mu_{t} \frac{\partial \kappa}{\partial x}\right)+\frac{\partial}{\partial y}\left(\mu_{t} \frac{\partial \kappa}{\partial y}\right)+\mu_{t} \Phi-\rho \varepsilon \\
\frac{\partial}{\partial x}\left(\rho V_{x} \varepsilon\right)+\frac{\partial}{\partial y}\left(\rho V_{y} \varepsilon\right)=\frac{\partial}{\partial x}\left(\frac{\mu_{t}}{\sigma_{\varepsilon}} \frac{\partial \varepsilon}{\partial x}\right)+\frac{\partial}{\partial y}\left(\frac{\mu_{t}}{\sigma_{\varepsilon}} \frac{\partial \varepsilon}{\partial y}\right)+C_{1 \varepsilon} \mu_{t} \frac{\varepsilon}{\kappa} \Phi-C_{2} \rho \frac{\varepsilon^{2}}{\kappa} \\
\mu_{e}=\mu+\mu_{t}, \mu_{t}=C_{\mu} \rho \frac{\kappa^{2}}{\varepsilon}, \Phi=2\left(\left(\frac{\partial V_{x}}{\partial x}\right)^{2}+\left(\frac{\partial V_{y}}{\partial y}\right)^{2}+\frac{1}{2}\left(\frac{\partial V_{y}}{\partial x}+\frac{\partial V_{x}}{\partial y}\right)^{2}\right)
\end{gathered}
$$


where $\rho$ - target air density ( $\rho=$ const $), V_{x}, V_{y}$ - fluid velocity vector projection, $\rho-$ fluid pressure, $\mu$ - given physical (laminar viscosity) ( $\mu=$ const $), \mu_{t}-$ turbulent (vortex) viscosity, $\mu_{e}$ - effective viscosity, $\kappa$ - kinetic energy of turbulence per unit mass of fluid, $\varepsilon$ - the rate of dissipation in heat of the kinetic energy of turbulence per unit volume of fluid, $C_{\mu}, C_{1 \varepsilon}, C_{2}, \sigma_{\varepsilon}-$ empirical constants of the $\kappa-\varepsilon$-model, $C_{\mu}=0,09, C_{1 \varepsilon}=1,44, C_{2}=1,92, \sigma_{\varepsilon}=1,3$.

Educations of continuity (1), motion (2) and equation of the $\kappa-\varepsilon$-model (3) recorded in a conservative manner; unknowns are functions $V_{x}, V_{y}, \rho, \kappa$ and $\varepsilon$.

The boundary conditions for the system of transport equations (1) - (3) can be:

- speed in case of speed setting at the border

- pressure. In the case of a free boundary, the pressure is set 0 relative to external pressure.

- adhesion conditions on the inner walls, the velocities are 0.

To obtain the air velocity fields in the body of the field stripper, this problem was solved numerically by the finite volume method.

To implement the numerical-analytical algorithm, the following boundary conditions were set - Figure 1:

1. In section 1-3, air is drawn in, the pressure is $0 \mathrm{~Pa}$. Here and below, the pressure is specified as the difference between atmospheric pressure.

2. In section 4-6, the air flow is released, the pressure is $0 \mathrm{~Pa}$.

3. In section $7,8-13$, the boundary conditions of the housing wall are set, the conditions for air adhesion - the flow velocity is $0 \mathrm{~m} / \mathrm{s}$.

4. The speed of rotation of the stripping drum is set. Initial speed $640 \mathrm{rpm}$ clockwise in the original coordinate system.

5. The rotation speed of the beater is set. Initial speed $2100 \mathrm{rpm}$ clockwise in the original coordinate system.

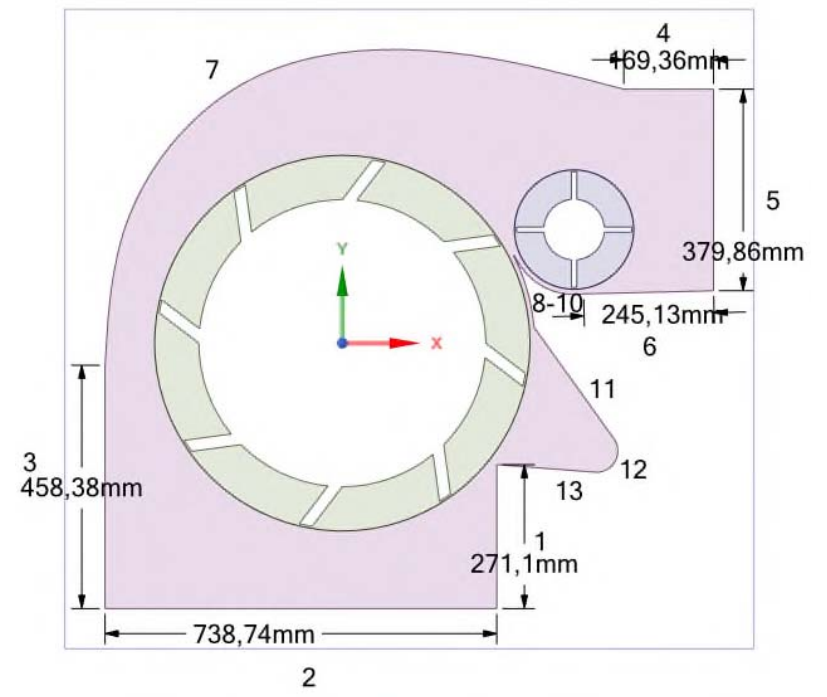

Fig. 1. Boundary conditions 
The drum and beater speeds have also been parameterized. The physical parameters of the air were set as follows:

- Density $-1.225 \mathrm{~kg} / \mathrm{m}^{3}$,

- Viscosity $-1.7910^{-5} \mathrm{~kg} /(\mathrm{m} \cdot \mathrm{s})$,

- Acceleration of gravity $9.81 \mathrm{~m} / \mathrm{s}^{2}$.

Numerical-analytical algorithm was implemented using two transport equations for turbulent characteristics (k- $\varepsilon$ model) [18].

\subsection{Mathematical model of the movement of a fragment of grain mass}

To describe the movement of a piece of grain mass in a field stripper, you can use different mechanical models (material point, absolutely rigid body, deformable body). In this work, we will consider a model of a material point. Within the framework of this model, we will represent a fragment of the grain mass in the form of a material point $M$. Mass of the point $M$ is equal $m$, the size of the fragment of the grain mass in comparison with the size of the installations is neglected.

Within the framework of these models, the movement of a fragment of the grain mass can be represented as the movement of a solid particle in an airflow.

Forces acting on a fragment of grain mass during movement:

1) Impact force of external force fields (gravity);

2) Forces due to non-uniform pressure distribution over the surface of a particle when it moves in a continuous flow:

- Archimedes force (at low air densities compared to grain density, the Archimedes force can be neglected);

- force caused by a change in pressure when the carrier air flow is accelerated or decelerated (for a small change in the relative flow velocity, it can be neglected);

- Zhukovsky's hydrodynamic force due to grain twisting by external forces;

- Magnus force due to the twisting of the grain by the carrying flow.

3) The force of the hydrodynamic effect of a flow moving at a certain speed relative to the grain, while the drag coefficient depends on the mode of motion and is determined by the Reynolds number;

4) Forces resulting from the interaction of a grain with a bounding surface;

5) Frictional forces at grain contact with the bounding surface.

When simulating the movement of a grain mass fragment in a field stripper, it is convenient to use a Cartesian coordinate system. At the same time, without loss of generality, the problem can be considered flat (Figure 2). The origin of the coordinate system is selected on the surface of the earth at the point where the sweep begins. 


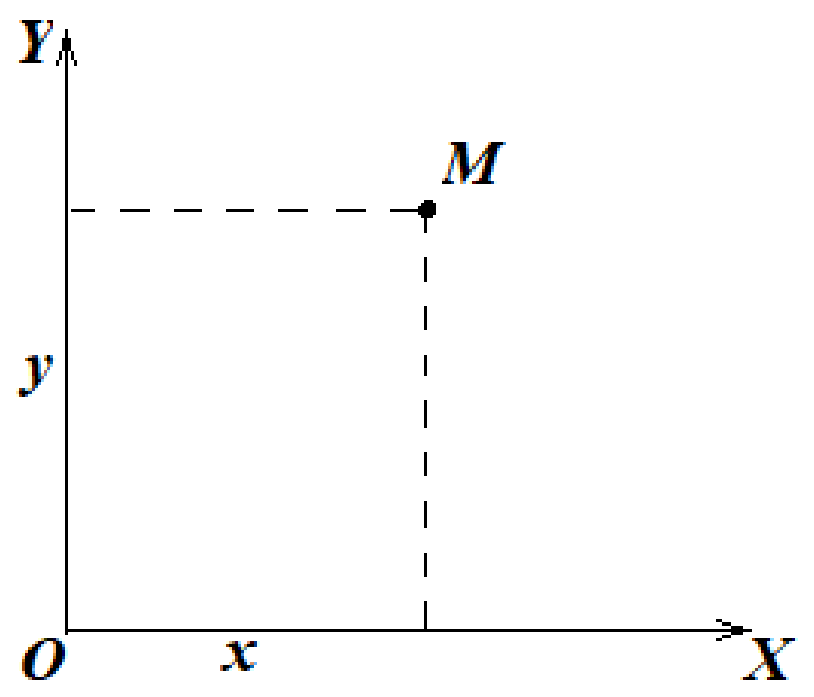

Fig. 2 Motion of the point $M$ in Cartesian coordinate system.

The motion of point $\mathrm{M}$ in this case will be described by the following system of differential equations [21]:

$$
\begin{aligned}
& m \ddot{x}=F_{x} \\
& m \ddot{y}=F_{y}
\end{aligned}
$$

where $m$ - grain mass, $F_{x}, F_{y}$-projection of resultant forces acting on the ear from the flowing air flow side, in its current position, $9.81 \mathrm{~m} / \mathrm{s}^{2}-$ acceleration of gravity. In the case when the particle moves in the air flow without contact with the surface of the chamber, the projections of the forces have the form

$$
\begin{gathered}
F_{x}=m \beta\left(V_{x}-\dot{x}\right) \\
F_{y}=-m g+m \beta\left(V_{y}-\dot{y}\right)
\end{gathered}
$$

where $V_{x}, V_{y}$ - corresponding components of the air flow rate in the installation chamber.

Since the quantities $V_{x}, V_{y}$ are set numerically as a result of calculating the air flow rates (1.1), then the solution of the system of differential equations (4) is carried out numerically in the software package of computer mathematics Maple.

When a fragment of grain mass moves in air, aerodynamic drag coefficient $\beta$ is determined experimentally. For this, a series of experiments was carried out on the fall of grain from different heights (Figure 3). 


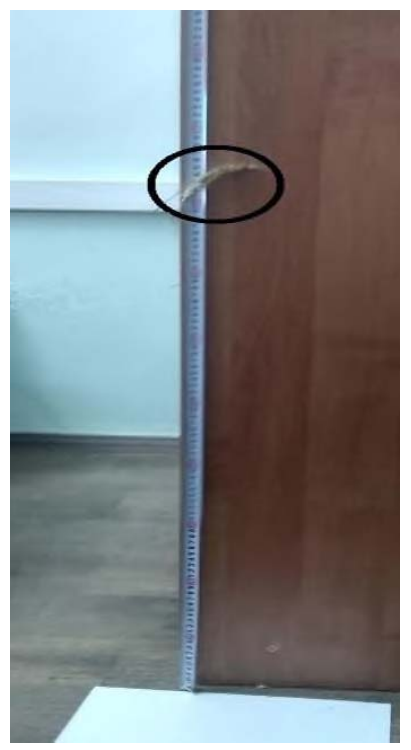

Fig. 3. Determination of the aerodynamic drag coefficient.

The drag coefficient can be found from the integration of the equation of rectilinear motion of a point ( $\mathrm{O} x$ axis pointing vertically down here):

$$
m \ddot{x}=m g-F_{c}
$$

Assuming at low speeds the aerodynamic drag force is proportional to the body mass and linearly dependent on the body speed $F_{c}=\beta m V$, we obtain by successive integration of the differential equation of motion (6):

$$
\begin{aligned}
& m \dot{V}=m g-m \beta V \\
& \dot{x}=g\left(1-e^{-\beta t}\right) \\
& x=g\left(t+\frac{e^{-\beta t}}{\beta}-\frac{1}{\beta}\right)
\end{aligned}
$$

where $x$-drop height, $t$ - drop time of the grain mass fragment. Into the equation (7) drop height and time values from a series of experiments are substituted, as a result of its numerical solution, the value of the coefficient $\beta$. The results of three series of experiments to determine the aerodynamic drag coefficient are presented in Table 1.

Table 1. Results of experiments to determine the coefficient of aerodynamic drag

\begin{tabular}{|c|c|c|c|}
\hline $\begin{array}{c}\text { № of experiments } \\
\text { series }\end{array}$ & Drop height $\boldsymbol{h}, \mathbf{m}$ & $\begin{array}{c}\text { Average flight } \\
\text { time } \boldsymbol{T}, \mathbf{s}\end{array}$ & $\begin{array}{c}\text { Drag coefficient, } \\
\boldsymbol{\beta}\end{array}$ \\
\hline 1 & 1 & 3,3 & 31.78 \\
\hline 2 & 1,5 & 4,9 & 31.92 \\
\hline 3 & 2 & 6,5 & 31.95 \\
\hline
\end{tabular}

As a result of a series of experiments, the arithmetic mean value $\beta=31.88$. 


\section{ANSYS Calculation of Airflow in a Field Stripper}

Figure 4 shows a finite-volume partition of the internal air domain of a field stripper.

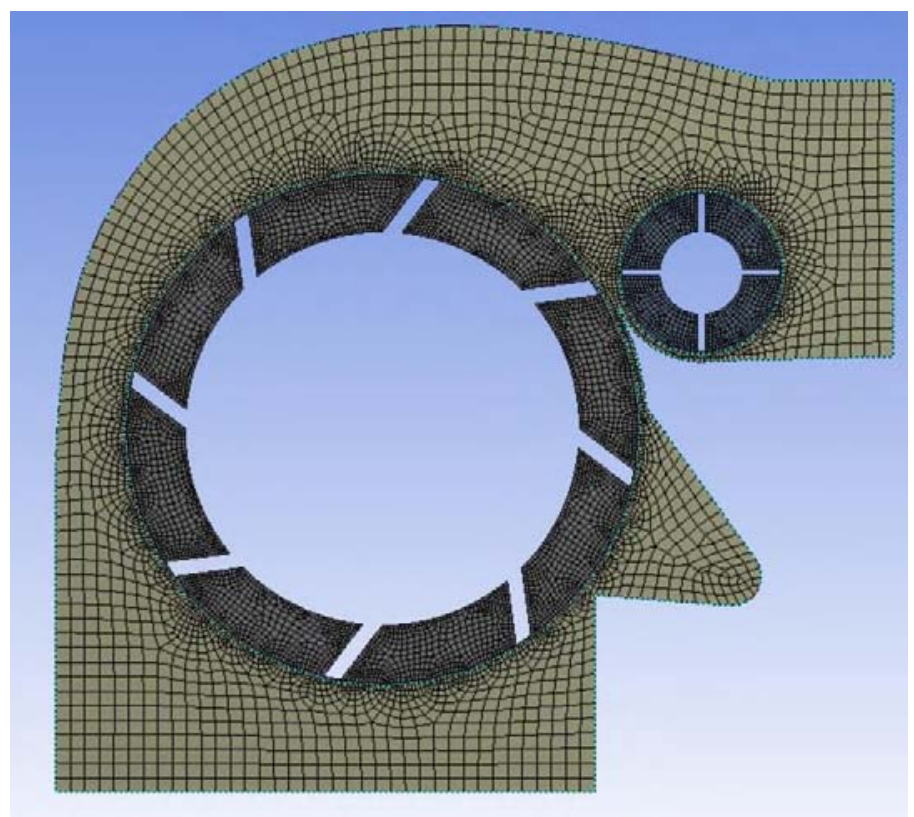

Fig. 4. Finite-volume partitioning.

\subsection{Calculation with standard parameters}

To obtain the distribution fields of flow rates in the field stripper body, a series of calculations was carried out at different geometric parameters and drum rotation speeds.

At Figures 5, 6 the contours of the velocity fields and the velocity vectors for the initial geometric parameters are shown (Figure 1). The rotation speeds of the drum and beater are $640 \mathrm{rpm}$ and $2100 \mathrm{rpm}$, respectively. The maximum design flow velocity was $36.2 \mathrm{~m} / \mathrm{s}$. On the left side of the drum, an increase in the flow rate is observed, due to the narrowing of the space between the drum and the wall of the housing. 


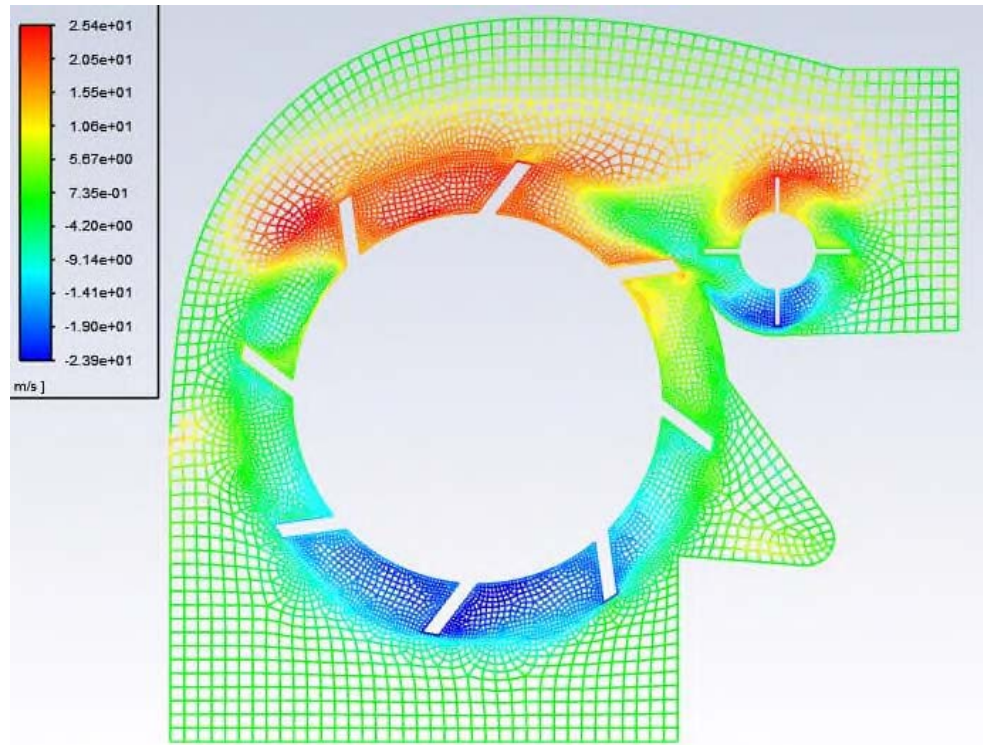

Fig. 5. Velocity vector contours for standard dimensions along the axis direction $X$

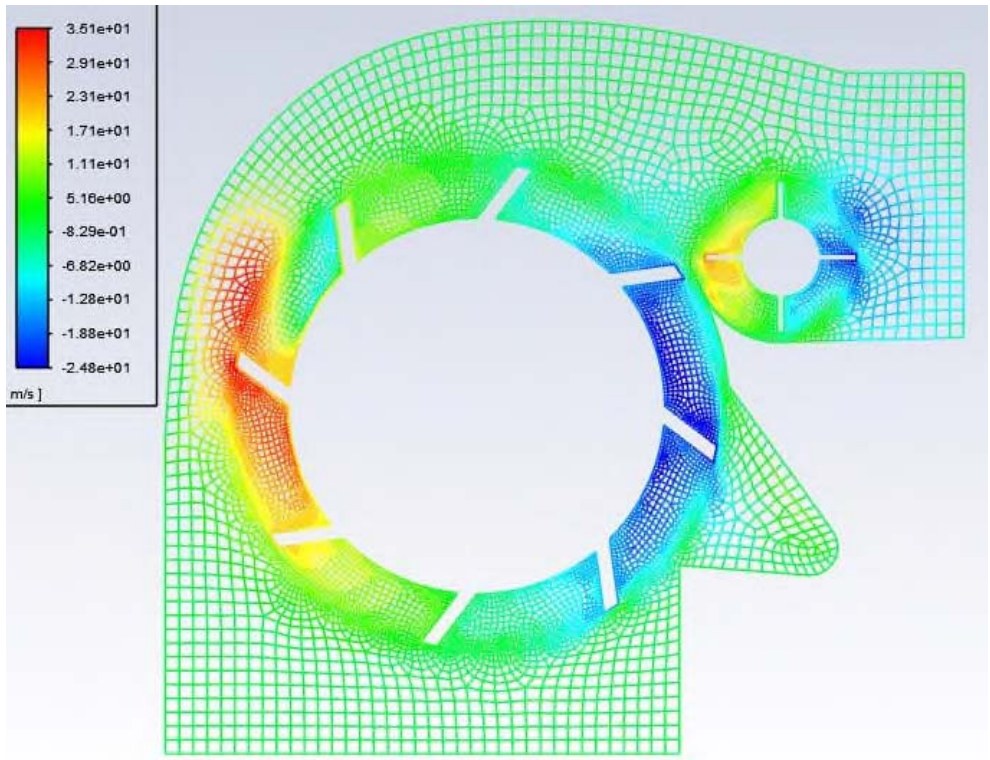

Fig. 6. Velocity vector contours for standard dimensions along the axis direction $\mathrm{Y}$

\subsection{Optimizing body geometry}

An optimization calculation was carried out to assess the effect of the hull geometry. The original body geometry (see Figure 1) has been changed by moving the top down $70 \mathrm{~mm}-$ Figure 7. 


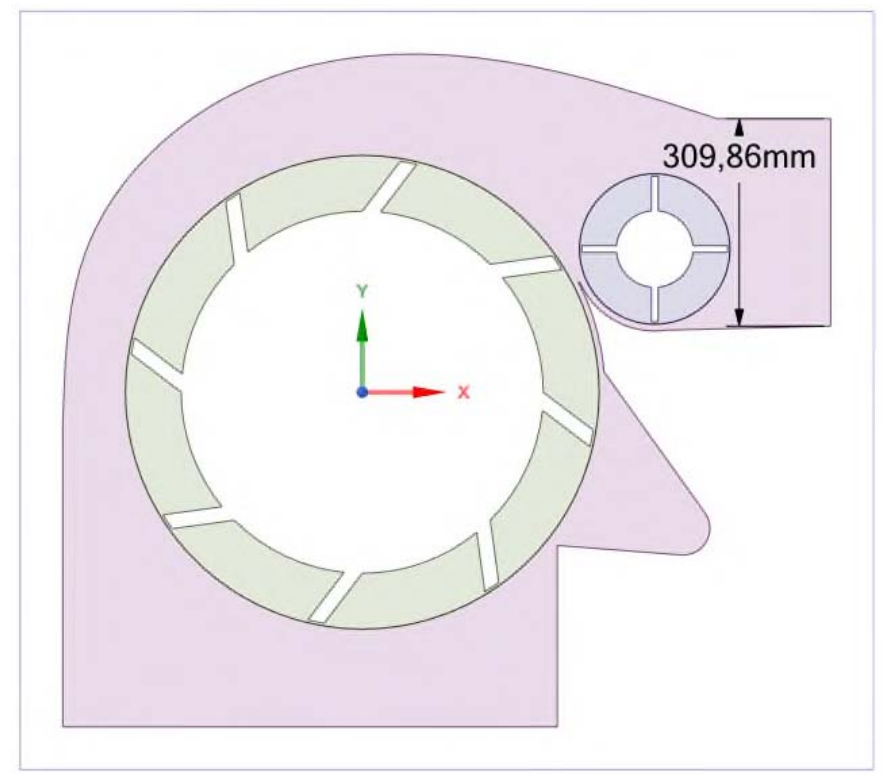

Fig. 7. Modified geometry of the original field stripper body.

Figures 8,9 show the contours of the velocity fields and the velocity vectors for the modified housing shown in Figure 9. The rotation speeds of the drum and beater are 640 $\mathrm{rpm}$ and $2100 \mathrm{rpm}$, respectively. The maximum design flow velocity was $31.2 \mathrm{~m} / \mathrm{s}$. For this geometry, a more even distribution of the air velocity fields is observed.

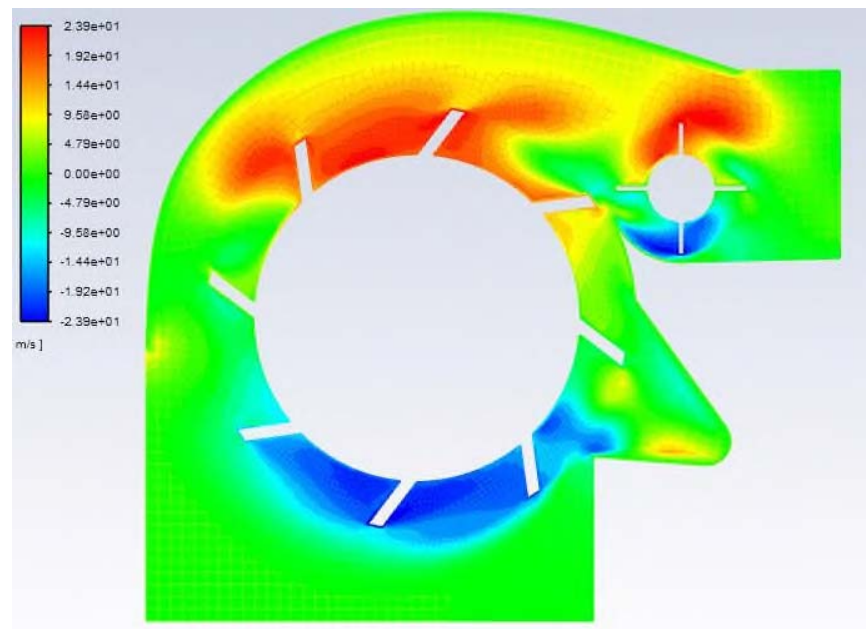

Fig. 8. Velocity contours for modified body geometry along the axis $X$. 


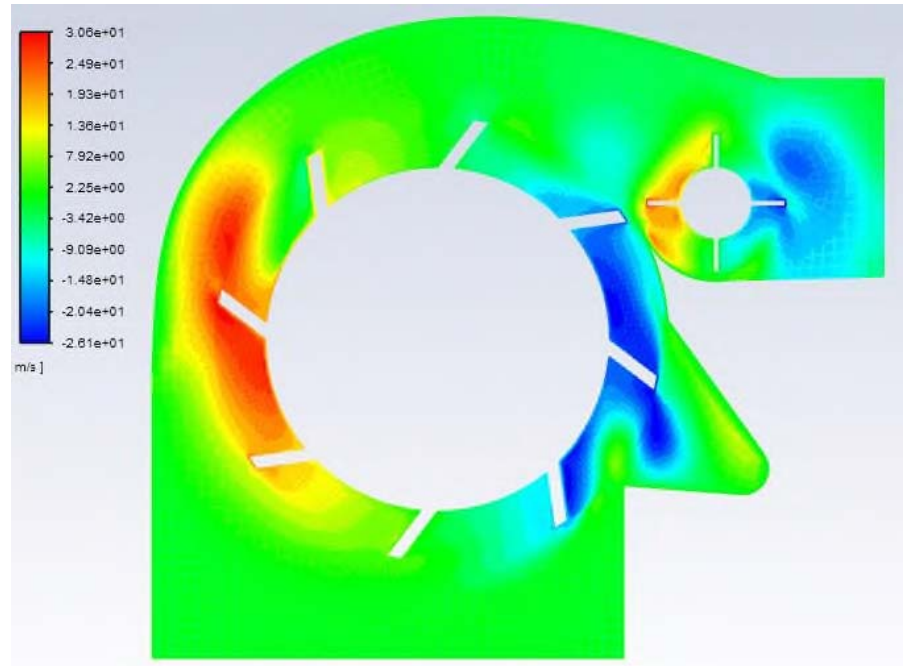

Fig. 9. Velocity contours for modified body geometry along the axis $Y$.

\subsection{Movement of a piece of grain mass in a field stripper}

To describe the movement of a grain mass fragment in a field stripper, a series of calculations was performed. At the initial moment of time, different initial coordinates of the grain mass fragment correspond to the random entry of the grain mass into the channel of the field stripper. Figures 10 and 11 show the trajectories of movement of a piece of grain mass from different initial positions for the optimized installation chamber (Figure 7).

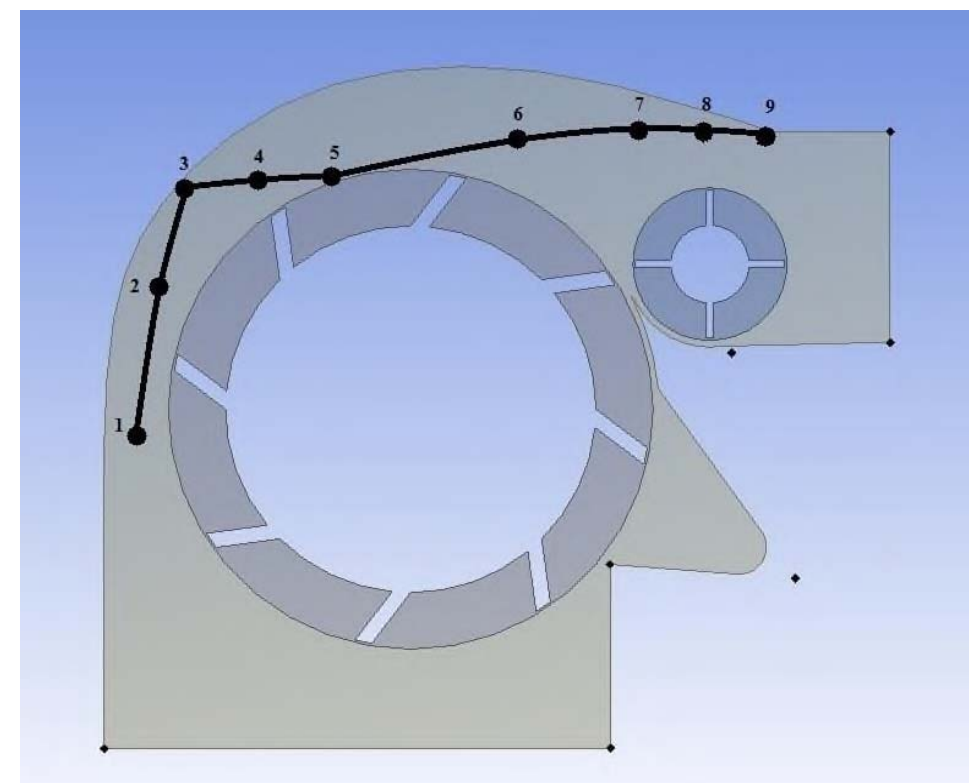

Fig. 10. Trajectory of movement of a fragment of grain mass. 


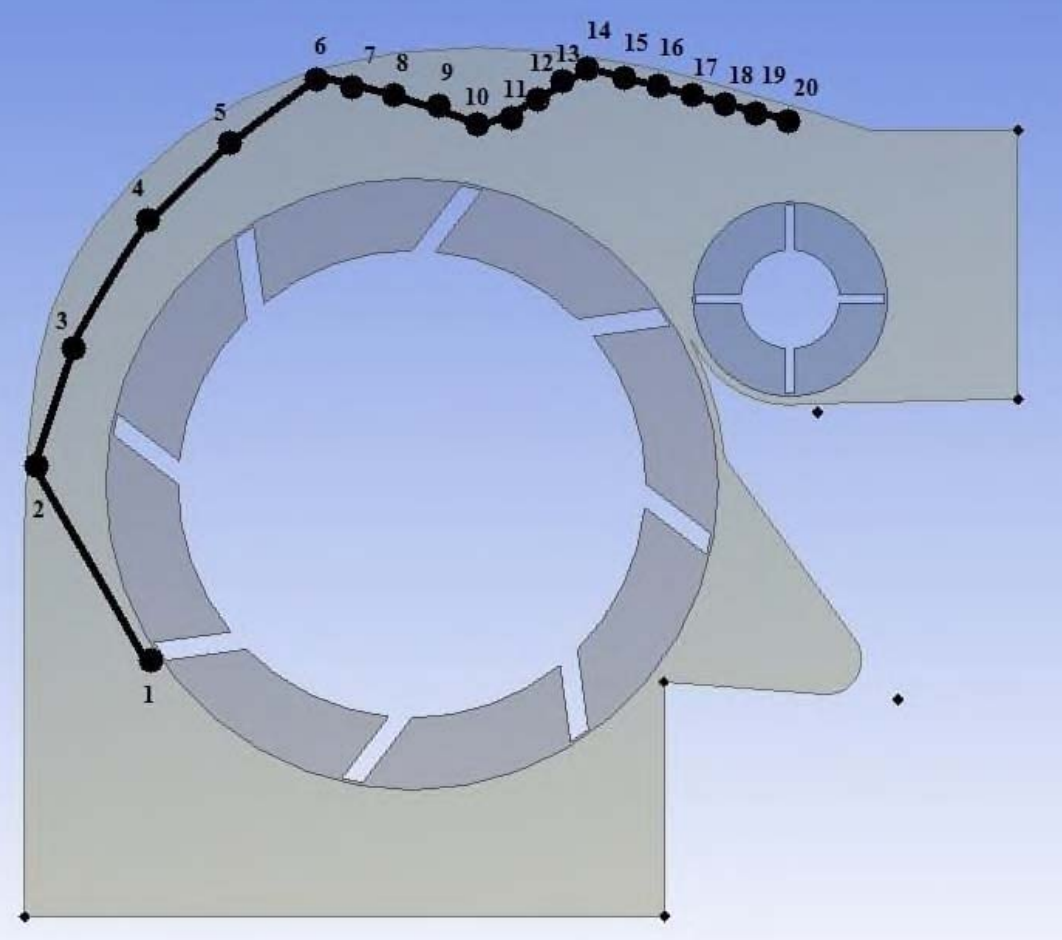

Fig. 11. Trajectory of movement of grain mass fragment.

In Fig. 10 it can be seen that the grain mass first hits the top deck, then against the rotating drum, and then, at the exit from the air channel, again against the deck. The recovery factor is taken to vary in the range from 0.8 to 1 . In Fig. 11, the grain mass is first picked up by the rotating drum, then it hits the top deck twice, and, entrained in the air flow, does not reach the drum, but again hits the deck.

Tables 2 and 3, respectively, show the values of coordinates and projections of speeds on the coordinate axes for different positions of the grain mass fragment. 
Table 2. Coordinates and speeds of the grain mass fragment.

\begin{tabular}{|c|c|c|c|c|}
\hline $\begin{array}{c}\text { Point } \\
\text { number in } \\
\text { figure }\end{array}$ & $\begin{array}{c}\text { Coordinate } \mathbf{x}, \\
\mathbf{m}\end{array}$ & $\begin{array}{c}\text { Coordinate y, } \\
\mathbf{m}\end{array}$ & $\begin{array}{c}\text { Speed } \\
\text { projection } \mathbf{~} \mathbf{x}, \\
\mathbf{m} / \mathbf{s}\end{array}$ & $\begin{array}{c}\text { Speed projection } \\
\mathbf{V y}, \mathbf{m} / \mathbf{s}\end{array}$ \\
\hline 1 & 10 & 45.8 & 1.39 & 23.45 \\
\hline 2 & 12 & 68 & 2.84 & 20.85 \\
\hline 3 & 14 & 80 & 17.0 & 3.00 \\
\hline 4 & 40 & 84 & 17.0 & -3.00 \\
\hline 5 & 42 & 85 & 23.0 & 3.00 \\
\hline 6 & 62 & 88 & 6.97 & 1.13 \\
\hline 7 & 77 & 90 & 6.97 & 1.13 \\
\hline 8 & 89 & 92 & 6.97 & 1.13 \\
\hline 9 & 97 & 94 & 6.97 & \\
\hline
\end{tabular}

Table 3. Coordinates and speeds of the grain mass fragment.

\begin{tabular}{|c|c|c|c|c|}
\hline $\begin{array}{c}\text { Point } \\
\text { number in } \\
\text { figure }\end{array}$ & $\underset{\mathbf{m}}{\text { Coordinate } x,}$ & $\underset{\mathrm{m}}{\text { Coordinate } \mathbf{y},}$ & $\begin{array}{c}\text { Speed } \\
\text { projection } V x, \\
\text { m/s }\end{array}$ & $\begin{array}{c}\text { Speed projection } \\
V y, \mathrm{~m} / \mathrm{s}\end{array}$ \\
\hline 1 & 0,15 & 0,32 & $-13,68$ & 17,96 \\
\hline 2 & 0.00 & 0,50 & $-10,3$ & 18,62 \\
\hline 3 & 0,09 & 0,68 & 9,44 & 16,59 \\
\hline 4 & 0,19 & 0,82 & 8,40 & 12,23 \\
\hline 5 & 0,26 & 0,93 & 6,10 & 8,81 \\
\hline 6 & 0,31 & 1,00 & 4,44 & 6,32 \\
\hline 7 & 0,37 & 0,98 & 5,70 & $-1,53$ \\
\hline 8 & 0,43 & 0,97 & 4,69 & $-1,19$ \\
\hline 9 & 0,51 & 0,96 & 4,15 & $-0,75$ \\
\hline 10 & 0,55 & 0,95 & 4,11 & $-0,09$ \\
\hline 11 & 0,59 & 0,95 & 3,73 & 0,40 \\
\hline 12 & 0,63 & 0,96 & 3,26 & 0,75 \\
\hline 13 & 0,66 & 0,97 & 2,92 & 1.00 \\
\hline 14 & 0,68 & 0,98 & 2,45 & 1,19 \\
\hline 15 & 0,70 & 0,99 & 2,68 & 1,11 \\
\hline 16 & 0,72 & 0,99 & 2,18 & $-0,20$ \\
\hline 17 & 0,73 & 0,99 & 1,58 & 0,15 \\
\hline 18 & 0,73 & 0,99 & 1,15 & 0.00 \\
\hline 19 & 0,73 & 0,99 & 0,83 & $-0,07$ \\
\hline 20 & 0,73 & 0,99 & 0,73 & $-0,07$ \\
\hline
\end{tabular}


The developed software makes it possible to determine not only the trajectory of movement of fragments of the grain mass, but also to calculate the speed of movement of the mass along the top deck.

\section{Conclusion}

The second stage of the iterative algorithm for solving the problem of optimizing the kinematic and geometric characteristics of the field stripper is developed. The task of studying the movement of the grain mass is divided into two stages. At the first stage, a model of the air mass movement in the considered installation is built and, on the basis of a mathematical model that takes into account the turbulence of the movement, the velocity and pressure field is calculated using the finite volume method. The optimization of the installation was carried out, as a result of which the shape of the surface of the upper deck was changed and a more uniform field of velocities was obtained in the area of movement of the grain mass. A mathematical model of the movement of a fragment of grain masses has been built and its parameters have been identified. A series of calculations was carried out according to the developed algorithm, the trajectories of the speed of movement of a fragment of grain mass were obtained, taking into account its interaction with the deck of the installation, on the basis of which its ribbing can be selected for resonant separation of grain from the ear.

\section{References}

1. R.S. Bello, Agricultural Machinery \& Mechanization 355 (Dominion Publishing Services, 2012)

2. A.A. Korotky, E.V. Marchenko, V.V. Ivanov, S.I. Popov, Ju.V. Marchenko, N.S. Dontsov, Model of forming vibration mechanochemical solid lubrication coating on surface of steel rope. XII International Scientific Conference on Agricultural Machinery Industry (INTERAGROMASH, 2019): IOP Conference Series: Earth and Environmental Science 403, 012116 (2019) doi:10.1088/1755-1315/403/1/012116.

3. V.V. Ivanov, S.I. Popov, N.S. Dontsov, G.E. Ekinil, Ju.A. Oleynikova, Ju.N. Denisenko, Mechanical coating formed under conditions of vibration exposure. XIII International Scientific and Practical Conference "State and Prospects for the Development of Agribusiness - INTERAGROMASH 2020»: E3S Web of Conferences 175, 05023 (2020) doi.org/10.1051/e3sconf/202017505023.

4. E.N. Ziborov, A.A. Matrosov, Calculation of strength characteristics and service life of the front axle of a combine State and prospects for the development of agricultural engineering: Proceedings of the 3rd international. scientific-practical Conf 199-200 (Rostov-on-Don, 2010)

5. D.A. Kolesnikov, A.A. Matrosov, Calculation of strength characteristics and service life of the rear axle of the combine. State and prospects for the development of agricultural engineering: Materials of the 3rd international. scientific-practical Conf. 200-202 (Rostov-on-Don, 2010)

6. A.A. Korotun, A.A. Matrosov, Calculation of strength characteristics and service life of the combine frame State and prospects for the development of agricultural engineering: Proceedings of the 3rd international. scientific-practical Conf. 217 (Rostov-on-Don, 2010)

7. O.I. Lysenok, A.A. Matrosov, Evaluation of the efficiency of the comb of the conveyor of the feeder chamber of the combine. State and prospects for the development of 
agricultural engineering: Proceedings of the 7th international. scientific-practical Conf. 86-87 (Rostov-on-Don, 2014)

8. Y.F. Lachuga, A.N. Soloviev, A.A. Matrosov, I.A. Panfilov, V.I. Pakhomov, D.V. Rudoy, Int. Conf. on Physics and Mechanics of New Materials and Their Applications (PHENMA) Hanoi, Hanoi University of Science and Technology 179-180 (2019)

9. E. Sokolova, V. Orobets, O. Sevostyanova, E. Gorchakov, D. Rudoy, A. Olshevskaya, A. Babajanyan, E3S Web of Conferences 175, 03015 (2020) https://doi.org/10.1051/e3sconf/202017503015.

10. M.A. Buryanov, Methods of mathematical modeling of the process of grain movement in the transporting channel of the stripping header, Tractors and agricultural machines 10, 27-30 (2015)

11. P.M. Parker, The 2021-2026 World Outlook for Agricultural Small Grain Headers for Combines Harvesting Machinery (Publisher: ICON Group International, Inc.) 315 (2020)

12. Y. Lachuga, A. Soloviev, A. Matrosov, I. Panfilov, V. Pakhomov, D. Rudoy, IOP Conf. Series: Earth and Environmental Science 403 (2019) 012055IOP Publishing doi:10.1088/1755-1315/403/1/012055

13. W.F. Ames, Numerical Methods for Partial Differential Equations, Publisher: Academic Press 357 (2014)

14. G.F. Pinder, Numerical Methods for Solving Partial Differential Equations: A Comprehensive Introduction for Scientists and Engineers, Publisher: Wiley 304 (2018)

15. F.R. Menter, The Scale-Adaptive Simulation Method for Unsteady Turbulent Flow Predictions. Pt. 1: Theory and Model Description. Journal Flow Turbulence and Combustion 85, 113-138 (2010)

16. S.S. Bayin, Essentials of Mathematical Methods in Science and Engineering, Publisher: John Wiley \& Sons, Inc. 960 (2019)

17. R. Wang, K. Kashinath, M. Mustafa, A. Albert, R. Yu, Towards Physics-informed Deep Learning for Turbulent Flow Prediction. ICLR 2020 Conference Blind Submission 13 (2019)

18. S. Mazumder, Numerical Methods for Partial Differential Equations: Finite Difference and Finite Volume Methods, Publisher: Academic Press 484 (2016)

19. Z. Li, Z. Qiao, T. Tang, Numerical Solution of Differential Equations: Introduction to Finite Difference and Finite Element Methods (Publisher: Cambridge University) 300 (2018)

20. J. Chaskalovic, Mathematical and Numerical Methods for Partial Differential Equations, Publisher: Springer 372 (2018)

21. R.M. Dreizler, C.S. Lüdde, Theoretical Mechanics, Publisher Springer 402 (2010) 